\title{
NOTE ON APPELL POLYNOMIALS
}

\section{M. SHEFFER}

An interesting characterization of Appell polynomials by means of a Stieltjes integral has recently been given by Thorne. ${ }^{1}$ We propose to give a second such representation, and to extend the result to the case of sets of polynomials of type zero, of which Appell sets form a subclass.

Appell sets may be defined by either of the following equivalent conditions: $\left\{P_{n}(x)\right\}, n=0,1, \cdots$, is an Appell set $\left(P_{n}\right.$ being of degree exactly $n$ ) if either

(i) $P_{n}^{\prime}(x)=P_{n-1}(x), n=1,2, \cdots$, or

(ii) there exists a formal power series $A(t)=\sum_{0}^{\infty} a_{n} t^{n}\left(a_{0} \neq 0\right)$ such that (again formally)

$$
A(t) e^{t x}=\sum_{0}^{\infty} P_{n}(x) t^{n}
$$

The function $A(t)$ may be called the determining function for the set $\left\{P_{n}(x)\right\}$. The essence of Thorne's result is the following:

Theorem of Thorne. A polynomial set $\left\{P_{n}(x)\right\}$ is an Appell set if and only if there exists a function $\alpha(x)$ of bounded variation on $(0, \infty)$ with the following properties:

(i) The moment integrals

$$
\mu_{n}=\int_{0}^{\infty} x^{n} d \alpha(x)
$$

all exist.

(ii) $\mu_{0} \neq 0$.

(iii) $\int_{0}^{\infty} P_{n}^{(r)}(x) d \alpha(x)=\delta_{n r}, \delta_{n r}=1$ for $n=r, \delta_{n r}=0$ for $n \neq r$. And for the set $\left\{P_{n}(x)\right\}$ the determining function $A(t)$ is given by

$$
A(t)=\left[\sum_{0}^{\infty} \mu_{n} \frac{t^{n}}{n !}\right]^{-1}=\left[\int_{0}^{\infty} e^{t x} d \alpha(x)\right]^{-1} .
$$

The Stieltjes integral characterization that we now give will be seen to be essentially different from that in (iii) above.

Theorem 1. A polynomial set $\left\{P_{n}(x)\right\}$ is an Appell set if and only

Presented to the Society, September 17, 1945; received by the editors May 10, 1945.

${ }^{1}$ C. J. Thorne, A property of Appell sets, Amer. Math. Monthly vol. 52 (1945) pp. 191-193. 
if there is a function $\beta(x)$ of bounded variation on $(0, \infty)$ with the following properties :

(i) The moment constants

$$
b_{n}=\int_{0}^{\infty} x^{n} d \beta(x)
$$

all exist.

(ii) $b_{0} \neq 0$.

(iii) For $n=0,1, \cdots$,

$$
P_{n}(x)=\int_{0}^{\infty} \frac{(x+t)^{n}}{n !} d \beta(t) .
$$

If (i) and (ii) hold, then $P_{n}(x)$ as given by (2) exists for each $n$, and is a polynomial of degree exactly $n$. Moreover, differentiation under the integral sign is permissible, so that $P_{n}^{\prime}(x)=P_{n-1}(x)$; that is, $\left\{P_{n}(x)\right\}$ is an Appell set.

Now suppose $\left\{P_{n}(x)\right\}$ is an Appell set, and let $A(t)$ be its determining function : $A(t)=\sum_{0}^{\infty} a_{n} t^{n}$. Define the sequence $\left\{b_{n}\right\}$ by

$$
b_{n}=n ! a_{n} \text {. }
$$

By a theorem of Boas $^{2}$ there is a function $\beta(x)$ of bounded variation on $(0, \infty)$ whose moment constants are $\left\{b_{n}\right\}$; and since $a_{0} \neq 0$, therefore $b_{0} \neq 0$. If we denote the right side of (2), which exists, by $Q_{n}(x)$, then $\left\{Q_{n}(x)\right\}$ is an Appell set. Since (formally)

$$
\sum Q_{n}(x) u^{n}=\int_{0}^{\infty} e^{u(x+t)} d \beta(t)=e^{u x} \int_{0}^{\infty} e^{u t} d \beta(t),
$$

the determining function for $\left\{Q_{n}(x)\right\}$ is

$$
\begin{aligned}
A^{*}(u)=\int_{0}^{\infty} e^{u t} d \beta(t) & =\sum_{0}^{\infty}\left\{\int_{0}^{\infty} t^{n} d \beta(t)\right\} \frac{u^{n}}{n !}=\sum_{0}^{\infty} b_{n} \frac{u^{n}}{n !} \\
& =\sum a_{n} u^{n}=A(u) .
\end{aligned}
$$

It follows that $\left\{Q_{n}(x)\right\} \equiv\left\{P_{n}(x)\right\}$, so that (2) holds.

COROLlaRY. The determining function for $\left\{P_{n}(x)\right\}$ is

$$
A(u)=\int_{0}^{\infty} e^{u t} d \beta(t)=\sum_{0}^{\infty} \frac{b_{n}}{n !} u^{n} .
$$

2 Widder, The Laplace transform, p. 139. This result of Boas is stated for real sequences, but it extends immediately to complex sequences. 
Let $\left\{I_{n}(x)\right\}$ be the Appell set

$$
I_{n}(x)=x^{n} / n !
$$

then (2) can be written

$$
P_{n}(x)=\int_{0}^{\infty} I_{n}(x+t) d \beta(t)
$$

Note that $I_{0}(0)=1, I_{n}(0)=0(n>0)$; it is this property that makes the determining function $A(t)$ so simply expressible in terms of the moment constants $\left\{b_{n}\right\}$. It is easily shown that in relation (5) the polynomials $I_{n}(x)$ can be replaced by any other Appell set, by a suitable change in the function $\beta(t)$. However, the determining function $A(t)$ is now not so easily expressed through the moment constants $\left\{b_{n}\right\}$. In fact, if $\left\{Q_{n}(x)\right\}$ is the Appell set defined by

$$
Q_{n}(x)=\int_{0}^{\infty} I_{n}(x+t) d \gamma(t)
$$

then

$$
P_{n}(x)=\int_{0}^{\infty} Q_{n}(x+t) d \beta(t)
$$

where the function $A(z)$ for $\left\{P_{n}(x)\right\}$ is given by

$$
A(z)=\int_{0}^{\infty} e^{z t} \int_{0}^{\infty} e^{z u} d \gamma(u) d \beta(t)=\sum_{0}^{\infty} a_{n} z^{n}
$$

with

$$
a_{n}=(1 / n !)\left\{c_{n} b_{0}+C_{n, 1} c_{n-1} b_{1}+\cdots+C_{n, n} c_{0} b_{n}\right\} .
$$

Here $\left\{b_{n}\right\},\left\{c_{n}\right\}$ are given by

$$
b_{n}=\int_{0}^{\infty} x^{n} d \beta(x), \quad c_{n}=\int_{0}^{\infty} x^{n} d \gamma(x), \quad n=0,1, \cdots .
$$

We turn now to sets of type zero. The polynomial set $\left\{P_{n}(x)\right\}$ is of type $z \mathrm{ero}^{3}$ if either of the following equivalent conditions holds:

(i) Formal series

$$
A(t)=\sum_{0}^{\infty} a_{n} t^{n} \quad\left(a_{0} \neq 0\right), \quad H(t)=\sum_{1}^{\infty} h_{n} t^{n} \quad\left(h_{1} \neq 0\right)
$$

3 I. M. Sheffer, Some properties of polynomial sets of type zero, Duke Math. J. vol. 5 (1939) pp. 590-622. 
exist for which (formally)

$$
A(t) e^{x H(t)}=\sum_{0}^{\infty} P_{n}(x) t^{n} .
$$

(ii) An operator $L[y(x)]$ of form

$$
L[y(x)] \equiv \sum_{1}^{\infty} l_{n} y^{(n)}(x) \quad\left(l_{1} \neq 0\right)
$$

exists such that

$$
L\left[P_{n}(x)\right]=P_{n-1}(x), \quad n=1,2, \cdots \text {. }
$$

If $L(t)$ is the series

$$
L(t)=\sum_{1}^{\infty} l_{n} t^{n},
$$

then $H(t)$ and $L(t)$ are (formally) inverse functions :

$$
L(H(t))=H(L(t))=t .
$$

All polynomial sets satisfying (14) will be said to be associated with the operator $L$. Sets associated with a given $L$ are distinguished one from another by their determining function $A(t)$ appearing in (12). Associated with a given $L$ there is a unique set $B_{L}=\left\{B_{n}(x)\right\}$ for which $B_{0}(0)=1, B_{n}(0)=0(n>0)$. This set we call the basic set for $L$; its determining function is $A(t) \equiv 1$.

THEOREM 2. In order that the polynomial set $\left\{P_{n}(x)\right\}$ be a set associated with the operator $L$ it is necessary and sufficient that there exist a function $\beta(x)$ of bounded variation on $(0, \infty)$, with the following properties:

(i) The moment constants $\left\{b_{n}\right\}$ for $\beta(x)$ all exist.

(ii) $b_{0} \neq 0$.

(iii) For $n=0,1, \cdots$,

$$
P_{n}(x)=\int_{0}^{\infty} B_{n}(x+t) d \beta(t)
$$

where $\left\{B_{n}(x)\right\}$ is the basic set for $L$.

Corollary. The determining function $A(t)$ for $\left\{P_{n}(x)\right\}$ has the expression

$$
A(t)=\int_{0}^{\infty} e^{u R(t)} d \beta(u)
$$


which is equivalent to

$$
A(L(z))=\int_{0}^{\infty} e^{u z} d \beta(u)=\sum_{0}^{\infty} \frac{b_{n}}{n !} z^{n} .
$$

If conditions (i), (ii) hold, it is clear that $\left\{P_{n}(x)\right\}$ as given by (17) is a set associated with the operator $L$. Now let $\left\{P_{n}(x)\right\}$ be a set relative to an operator $L$; to show that (i), (ii), (iii) hold. Let $A(t)$ be the determining function for $\left\{P_{n}(x)\right\}$, so that we have relation (12). Since $A$ and $L$ are given, the function $A(L(z))$ is known; let its (formal) power series be

$$
A(L(z))=\sum_{0}^{\infty} a_{n}^{*} z^{n}
$$

Now define $\left\{b_{n}\right\}$ so that $b_{n}=n ! a_{n}^{*}$, and let $\beta(t)$ be the function of bounded variation on $(0, \infty)$, guaranteed by the Boas theorem, whose moments are $\left\{b_{n}\right\}$. With this $\beta(t)$, the right side of (17) defines a polynomial set $\left\{Q_{n}(x)\right\}$, associated with $L$, whose determining function is seen to be the function $A(t)$ given by (18). As (18) is carried into (19) by the transformation $z=H(t)$ (and therefore $t=L(z)$ ), it follows that $\left\{P_{n}\right\},\left\{Q_{n}\right\}$ have the same determining function $A(t)$, and are therefore the same set. Hence (17) holds.

In Theorem 2 the representation (17) can be replaced by

$$
P_{n}(x)=\int_{0}^{\infty} Q_{n}(x+t) d \beta(t)
$$

where $\left\{Q_{n}(x)\right\}$ is any polynomial set associated with $L$; but in this case the determining function $A(t)$ for $\left\{P_{n}(x)\right\}$ has a more complicated representation than (18) or (19). In fact, if we write

$$
Q_{n}(x)=\int_{0}^{\infty} B_{n}(x+t) d \gamma(t)
$$

it is readily shown that

$$
A(t)=\int_{0}^{\infty} \int_{0}^{\infty} e^{(u+w) H(t)} d \gamma(u) d \beta(w),
$$

or, what is equivalent to this,

$$
A(L(z))=\int_{0}^{\infty} \int_{0}^{\infty} e^{(u+w) z} d \gamma(u) d \beta(w)=\sum_{0}^{\infty} a_{n}^{*} z^{n},
$$

where

$$
a_{n}^{*}=(1 / n !)\left\{c_{n} b_{0}+C_{n, 1} c_{n-1} b_{1}+\cdots+C_{n, n} c_{0} b_{n}\right\}
$$


Here $\left\{b_{n}\right\},\left\{c_{n}\right\}$ are the moment constants for $\beta(t), \gamma(t)$ respectively. We close with two observations.

REMARK 1. The Thorne theorem carries over to polynomial sets of type zero; we have only to replace the condition (iii) of that theorem by

$$
\int_{0}^{\infty} L^{(r)}\left[P_{n}(x)\right] d \alpha(x)=\delta_{n r}
$$

where $L^{(1)}=L, L^{(2)}, \ldots$ are the iterates of $L$. Also, the determining function $A(t)$ for $\left\{P_{n}(x)\right\}$ is given by

$$
A(t)=\left[\int_{0}^{\infty} e^{x H(t)} d \alpha(x)\right]^{-1},
$$

or, by the equivalent expression,

$$
A(L(z))=\left[\int_{0}^{\infty} e^{x z} d \alpha(x)\right]^{-1}=\left[\sum_{0}^{\infty} \frac{\mu_{n}}{n !} z^{n}\right]^{-1} .
$$

REMARK 2. Throughout this note we have not hesitated to use formal power series. This does not invalidate the results obtained; it has permitted the results to be gotten faster. As to how we could have reasoned without the use of these formal series, we refer to the reference in footnote 3, where there is to be found on page 596 a note of justification.

The Pennsyluania State College 